\title{
Regulation of the Pcol/PcoR quorum-sensing system in Pseudomonas fluorescens 2P24 by the PhoP/PhoQ two-component system
}

Correspondence

Li-Qun Zhang

zhanglq@cau.edu.cn

Received 22 May 2008

Revised 5 October 2008

Accepted 7 October 2008

\author{
Qing Yan, Wei Gao, Xiao-Gang Wu and Li-Qun Zhang
}

Department of Plant Pathology, China Agricultural University, Beijing 100193, China

\begin{abstract}
A quorum-sensing locus, $p c o l / p c o R$, which is involved in the regulation of root colonization and plant disease-suppressive ability, was previously identified in Pseudomonas fluorescens 2P24. In this study, we performed random mutagenesis using mini-Tn5 in order to screen the upstream transcriptional regulators of $\mathrm{pcol}$, a biosynthase gene responsible for the synthesis of $\mathrm{N}$ acylhomoserine lactone signal molecules. Two mutants, PM400 and PM410, with elevated pcol gene promoter activity, were identified from $\sim 10000$ insertion clones. The amino acid sequences of the interrupted genes in these two mutants were highly similar to $\mathrm{PhoQ}$, a sensor protein of the two-component regulatory system $\mathrm{PhoP} / \mathrm{PhoQ}$, which responds to environmental $\mathrm{Mg}^{2+}$ starvation and regulates virulence in Salmonella typhimurium and antimicrobial peptide resistance in Pseudomonas aeruginosa. The promoter activity of $p c o l$ was also induced under $10 \mathrm{w}-\mathrm{Mg}^{2+}$ conditions in the 2P24 strain of $P$. fluorescens. Deletion mutagenesis and complementation experiments demonstrated that the transcription of $p c o l$ was negatively regulated by the sensor PhoQ but positively regulated by the response regulator PhoP. Genetic evidence also indicated that transcription of the outer-membrane protein gene oprH was induced by $\mathrm{Mg}^{2+}$ starvation through regulation of the wild-type PhoP/PhoQ system. Additionally, PhoQ was involved in biofilm formation by $2 \mathrm{P} 24$ under low- $\mathrm{Mg}^{2+}$ conditions through a PhoP-independent pathway.
\end{abstract}

\section{INTRODUCTION}

Quorum sensing (QS) is a widespread mechanism of cell-tocell communication used by many bacteria to monitor their population densities and to adapt their behaviours by regulating gene expression in response to fluctuations in cell population density (for reviews see Miller \& Bassler, 2001; Keller \& Surette, 2006). QS plays an important role in a diverse array of physiological activities, including symbiosis, virulence, competence, conjugation, antibiotic production, swarming, sporulation and biofilm formation (González \& Keshavan, 2006; de Kievit \& Iglewski, 2000; Sakuragi \& Kolter, 2007; Waters et al., 2008). The regulation of QS has been intensively studied in recent years (Venturi, 2006). In pseudomonads, identified regulatory elements of QS include the stationary-phase sigma factor RpoS (Bertani \& Venturi, 2004), the two-component regulatory system GacS/GacA (Reimmann et al., 1997), the small RNA-binding regulator RsmA (Pessi et al., 2001), the LuxR family member VqsR

Abbreviations: AHL, $\mathrm{N}$-acylhomoserine lactone; PVC, polyvinyl chloride; OS, quorum sensing.

The GenBank/EMBL/DDBJ accession number for the DNA sequence containing the oprH, phoP and phoQ genes of $P$. fluorescens 2P24 is EU569830.

Supplementary material is available with the online version of this paper.
(Juhas et al., 2004) and the tetrahelical H-T-H superclass member RsaL (Rampioni et al., 2007). The discovery of new regulators of QS will help to further elucidate the signal transduction mechanism in bacteria that survive under various environmental conditions.

The two-component PhoP/PhoQ system was first recognized in Salmonella typhimurium (Kier et al., 1979) as a regulatory system that responds to extracellular $\mathrm{Mg}^{2+}$ starvation (Soncini et al., 1996; Véscovi et al., 1996). PhoQ is a bifunctional sensor protein that detects environmental changes in $\mathrm{Mg}^{2+}$ concentration and, by modulating its phosphatase/kinase activity, defines the phosphorylation state of the regulator PhoP (Castelli et al., 2000). Multiple studies have indicated that the PhoP/PhoQ system is a master regulator of virulence in $S$. typhimurium (for a review see Groisman, 2001). Recent studies on PhoP/PhoQ in Pseudomonas aeruginosa PAO1 have revealed that this system plays an important role in the expression of the outer-membrane protein OprH (Macfarlane et al., 1999), the modification of lipid A (Ernst et al., 1999) and the resistance to cationic antimicrobial peptides and aminoglycosides (Macfarlane et al., 2000). It has also been demonstrated to regulate several virulence determinants, including swarming, lipase production and virulence in a neutropenic mouse model (Brinkman et al., 2001). 
In the present study, a random mini-Tn5 mutagenesis procedure was used to identify the upstream regulator(s) of QS in Pseudomonas fluorescens 2P24, a plant-diseasesuppressive bacterium isolated from the wheat rhizosphere (Wei \& Zhang, 2006). The Tn5-interrupted gene exhibited high amino acid similarities to the $p h o Q$ gene of several bacteria and the results indicated that the $\mathrm{PhoP} / \mathrm{PhoQ}$ system may be an important upstream regulator of QS in $P$. fluorescens $2 \mathrm{P} 24$.

\section{METHODS}

Bacterial strains, plasmids and growth conditions. The strains and plasmids used in this study are listed in Table 1. P. fluorescens 2P24 and its derivatives were grown in Luria-Bertani (LB) or ABM minimal medium (Chilton et al., 1974) at $28{ }^{\circ} \mathrm{C}$. To study the effect of $\mathrm{Mg}^{2+}$ concentration, LB medium containing $30 \mathrm{mM} \mathrm{NaCl}$ (low$\mathrm{Mg}^{2+}$ condition) or $15 \mathrm{mM} \mathrm{MgCl}$ (high- $\mathrm{Mg}^{2+}$ condition) (see Supplementary Fig. S1, available with the online version of this paper), and $\mathrm{ABM}$ minimal medium containing $0.1 \mathrm{mM} \mathrm{MgSO}_{4}$ (low$\mathrm{Mg}^{2+}$ condition) or $15 \mathrm{mM} \mathrm{MgSO} 4$ (high- $\mathrm{Mg}^{2+}$ condition) were used. When required, the growth media were supplemented with ampicillin $\left(50 \mu \mathrm{g} \mathrm{ml}^{-1}\right)$, kanamycin $\left(50 \mu \mathrm{g} \mathrm{ml}^{-1}\right)$, tetracycline $(20 \mu \mathrm{g}$ $\left.\mathrm{ml}^{-1}\right)$, or X-Gal $\left(40 \mu \mathrm{g} \mathrm{ml}^{-1}\right)$.
DNA manipulations. Plasmid DNA extractions and other molecular assays were performed according to standard procedures (Sambrook et al., 1989). Nucleotide sequencing was performed by Sun Biotechnology. Nucleotide and deduced amino acid sequences were analysed using the National Center for Biotechnology Information BLAST server (http://www.ncbi.nlm.nih.gov).

Tn5 mutagenesis and cloning of oprH, phoP and phoQ. In order to identify the potential upstream regulators of pcoI gene transcription, we randomly mutated a 2P24 reporter strain, PM101 ( $p c o I::$ lac $Z$ ), using a mini-Tn5 mutagenesis procedure according to a previously described method (Herrero et al., 1990). The mutated cells were incubated on ABM plates containing ampicillin, kanamycin and the $\beta$-galactosidase substrate X-Gal at $28{ }^{\circ} \mathrm{C}$ for $30 \mathrm{~h}$. Colonies with increased $\beta$-galactosidase activity (indicated by a more intense blue colour) were selected and purified. The mini-Tn5-disrupted genes in these mutants were identified by cloning and sequencing the genomic DNA fragments flanking the transposon. Using primers phoQT3/phoQT7 (see Supplementary Table S1, available with the online version of this paper, for details of primers), which were designed according to the flanking sequence, three cosmids (p18-25, p22-31and p28-18) were screened out by PCR from the genomic DNA library of 2P24 (Wei \& Zhang, 2005). A $5.7 \mathrm{~kb}$ HindIII fragment from p18-25 was subcloned into pBluescript II SK $(+)$, giving rise to the plasmid pBS-6Q. Sequencing of this fragment identified the entire gene sequences of $o p r H$, phoP and $p h o Q$ (Fig. 1a).

Table 1. Bacterial strains and plasmids used in this study.

$\mathrm{Ap}^{\mathrm{r}}, \mathrm{Gm}^{\mathrm{r}}, \mathrm{Km}^{\mathrm{r}}, \mathrm{Sm}^{\mathrm{r}}$ and $\mathrm{Tc}^{\mathrm{r}}$ indicate resistance to ampicillin, gentamicin, kanamycin, streptomycin and tetracycline, respectively

\begin{tabular}{|c|c|c|}
\hline Strain or plasmid & Description & Reference or source \\
\hline \multicolumn{3}{|l|}{ P. fluorescens } \\
\hline $2 \mathrm{P} 24$ & Wild-type, $A^{r}{ }^{r}$ & Wei \& Zhang (2005) \\
\hline PM101 & pcoI: : lacZ reporter fusion of $2 \mathrm{P} 24, \mathrm{Ap}^{\mathrm{r}}$ & Yan et al. (2008) \\
\hline PM400, PM410 & phoQ:: Tn5 in PM101, $\mathrm{Ap}^{\mathrm{r}} \mathrm{Km}^{\mathrm{r}}$ & This study \\
\hline PM401 & phoQ in-frame deletion in PM101, $\mathrm{Ap}^{\mathrm{r}}$ & This study \\
\hline PM402 & phoQ in-frame deletion in $2 \mathrm{P} 24, \mathrm{Ap}^{\mathrm{r}}$ & This study \\
\hline PM403 & phoP in-frame deletion in PM101, $\mathrm{Ap}^{\mathrm{r}}$ & This study \\
\hline PM404 & phoP in-frame deletion in $2 \mathrm{P} 24, \mathrm{Ap}^{\mathrm{r}}$ & This study \\
\hline \multicolumn{3}{|l|}{ A. tumefaciens } \\
\hline NTL4(pZLR4) & $\operatorname{traG}:$ : lacZ, AHL indicator, $\mathrm{Gm}^{\mathrm{r}}$ & Cha et al. (1998) \\
\hline \multicolumn{3}{|l|}{ Plasmids } \\
\hline pBluescript II SK $(+)$ & Cloning vector, $\mathrm{Ap}^{\mathrm{r}}$ & Stratagene \\
\hline pHSG299 & ColE1 origin, cloning vector, $\mathrm{Km}^{\mathrm{r}}$ & TaKaRa \\
\hline pRK415 & Escherichia-Pseudomonas shuttle vector, $\mathrm{Tc}^{\mathrm{r}}$ & Keen et al. (1988) \\
\hline p18-25 & Cosmid clone containing entire phoQ gene, $\mathrm{Tc}^{\mathrm{r}}$ & This study \\
\hline pBS-6Q & $\begin{array}{l}5.7 \mathrm{~kb} \text { HindIII fragment from p18-25 containing entire } o p r H \text {, phoP and } \\
\text { phoQ cloned into pBluescript II SK }(+)\end{array}$ & This study \\
\hline pUTKm & Delivery plasmid for Tn5; R6K replicon, $\mathrm{Ap}^{\mathrm{r}} \mathrm{Km}^{\mathrm{r}}$ & Herrero et al. (1990) \\
\hline pRG970 & $\begin{array}{l}\text { Cloning vector containing promoterless lacZYA for construction of } \\
\text { transcriptional fusions, } \mathrm{Sm}^{\mathrm{r}}\end{array}$ & Van den Eede et al. (1992) \\
\hline pRG970Km & A derivative plasmid of $\mathrm{pRG} 970 \mathrm{~b}, \mathrm{Km}^{\mathrm{r}}$ & This study \\
\hline p970-pcoI & pRG970Km containing pcoI: : lacZ transcriptional fusion, $\mathrm{Km}^{\mathrm{r}}$ & This study \\
\hline p970-H & pRG970Km containing oprH: : lacZ transcriptional fusion, $\mathrm{Km}^{\mathrm{r}}$ & This study \\
\hline p299 2 phoP & Suicide plasmid containing deleted phoP gene on pHSG299, $\mathrm{Km}^{\mathrm{r}}$ & This study \\
\hline p299 2 phoQ & Suicide plasmid containing deleted phoQ gene on pHSG299, $\mathrm{Km}^{\mathrm{r}}$ & This study \\
\hline p415-phoP & $\begin{array}{l}\text { phoP, as a } 2.2 \mathrm{~kb} N c o \mathrm{I}-P s t \mathrm{I} \text { fragment from pBS-6Q, was cloned downstream } \\
\text { of the lac promoter in pRK415, } \mathrm{Tc}^{\mathrm{r}}\end{array}$ & This study \\
\hline p415-phoQ & $\begin{array}{l}\text { phoQ, as a } 1.9 \mathrm{~kb} \text { Sall-EcoRV fragment from pBS-6Q, was cloned downstream } \\
\text { of the lac promoter in pRK415, Tc }{ }^{\mathrm{r}}\end{array}$ & This study \\
\hline
\end{tabular}




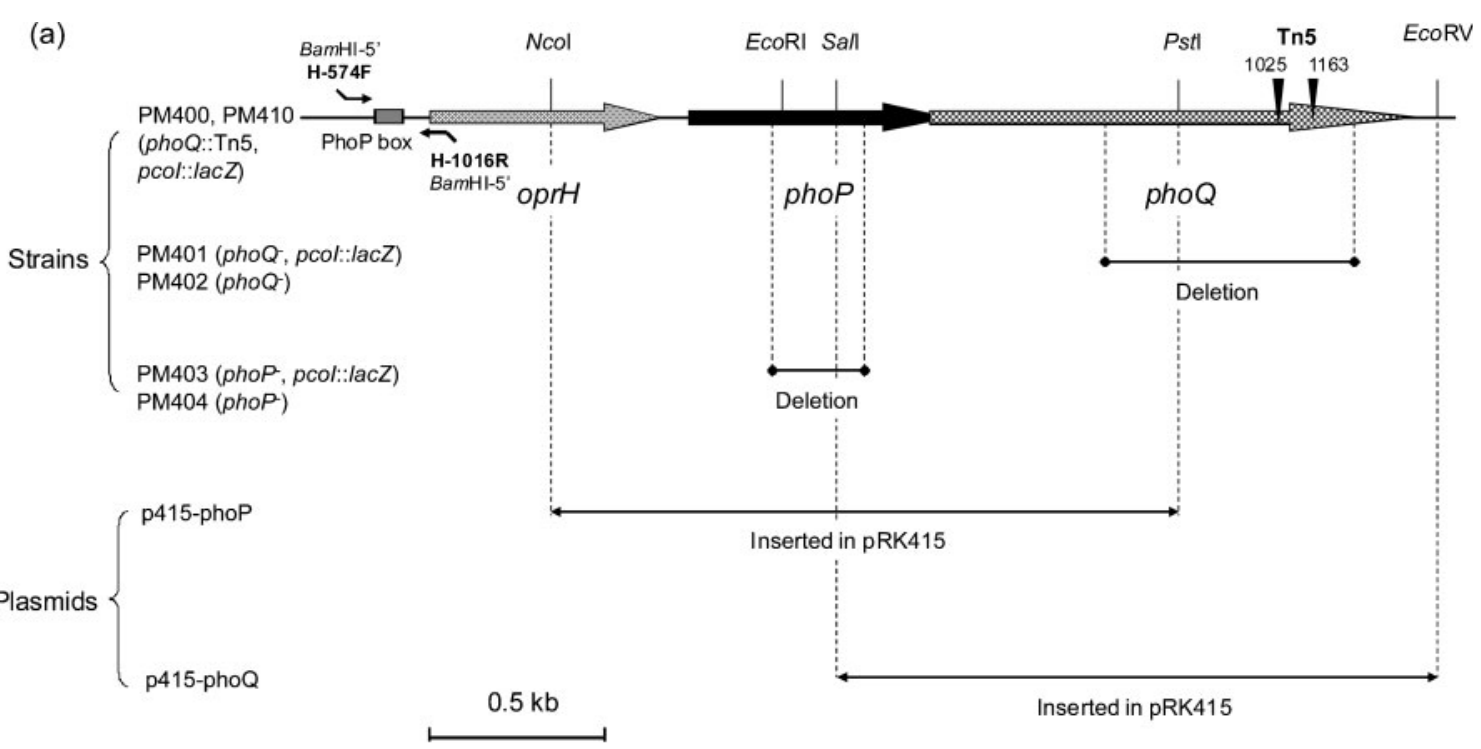

(b)

1 TAAAAATGGTCCGCTCAGATTAGCCGATTCATTCAGCGCAGgTTCAGAGAACGTTCAAG

61 GGGGGTTCAGGGAGCGCTGGTTAACGTGAAAGCGACTTCACACACCACCCACGTTGCAA

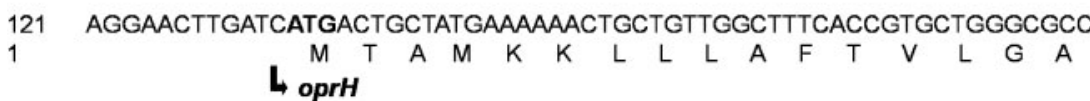

Fig. 1. (a) Schematic diagram of $P$. fluorescens $2 \mathrm{P} 24$ oprH, phoP and phoQ genes. The single-headed arrows represent the location and orientation of the genes in the $P$. fluorescens 2P24 chromosome. The main restriction enzyme sites are shown. Tn5 in PM400 and PM410 was inserted in the phoQ gene at position $1025 \mathrm{bp}$ and $1163 \mathrm{bp}$, respectively. The construction of strains PM400-PM404 and of plasmids p415-phoP and p415-phoO is described in Methods. 574F and 1016R (Table S1) indicate the primers used in the PCR to construct the reporter plasmid p970-H. (b) Upstream nucleotide sequence and part of the translated oprH gene in P. fluorescens 2P24. The start of oprH is labelled. The hexanucleotide direct repeats (PhoP box) upstream of $\mathrm{oprH}$ are indicated by underlines.

The promoter region, including the proposed PhoP box and the upstream fragment of oprH (Fig. 1), was amplified by PCR using primers $\mathrm{H}-574 \mathrm{~F}$ and $\mathrm{H}-1016 \mathrm{R}$ (Table S1), and a $442 \mathrm{bp} \mathrm{BamHI}$ fragment was cloned ahead of a promoterless lac $Z$ in $\mathrm{p} 970 \mathrm{Km}$, a derivative plasmid of pRG970 (Van den Eede et al., 1992). The resulting plasmid, $\mathrm{p} 970-\mathrm{H}$, was used to examine the activity of the oprH promoter.

Construction of phoP and phoQ in-frame deletion mutants. phoP and $p h o Q$ deletion mutants were constructed using a two-step homologous recombination strategy. The detailed protocol and PCR primers (Table S1) are given in the online supplementary material. The in-frame deletion structures of $p h o P$ and $p h o Q$ were ligated into

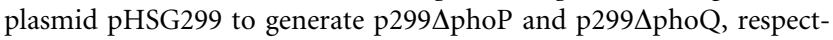

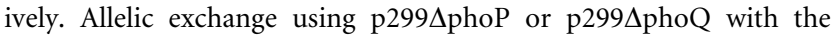
wild-type 2P24 resulted in mutants PM404 and PM402, respectively;

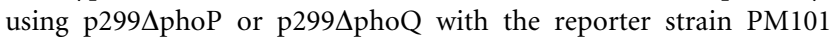
(pcoI::lacZ) resulted in mutants PM403 and PM401, respectively (Fig. 1a).

In order to construct a complementary plasmid, the phoP gene was inserted as a $2.2 \mathrm{~kb} \mathrm{NcoI-PstI} \mathrm{fragment} \mathrm{into} \mathrm{the} \mathrm{shuttle} \mathrm{vector}$ pRK415 to create p415-phoP. Similarly, the phoQ gene was inserted as a $1.9 \mathrm{~kb}$ SalI-EcoRV fragment into pRK415 to create p415-phoQ (Fig. 1a).

Isolation and detection of $\boldsymbol{N}$-acylhomoserine lactone (AHL). $P$. fluorescens 2P24 and its derivatives were grown in LB liquid medium at $28{ }^{\circ} \mathrm{C}$ for $36 \mathrm{~h}$. Aliquots $(0.8 \mathrm{ml})$ of the cultures were extracted with the same volume of ethyl acetate; the extracts were then dried and resuspended in $0.1 \mathrm{ml}$ methanol. For quantitative analysis of AHL, $3 \mu \mathrm{l}$ aliquots of the samples were incubated with $0.2 \mathrm{ml}$ of the AHL biosensor Agrobacterium tumefaciens NTL4(pZLR4) (Cha et al., 1998) $\left(\mathrm{OD}_{600} 0.8\right)$. The reaction mixture was incubated at $28{ }^{\circ} \mathrm{C}$ for $3 \mathrm{~h}$ and the $\beta$-galactosidase activity of the biosensor cells was assayed (see below).

$\boldsymbol{\beta}$-Galactosidase assay and statistical analysis. For $\beta$-galactosidase measurement, Pseudomonas and Agrobacterium strains that contained $\beta$-galactosidase reporter genes were grown in LB and $\mathrm{ABM}$ liquid medium, respectively, at $28{ }^{\circ} \mathrm{C}$ with shaking at 130 r.p.m. in $50 \mathrm{ml}$ flasks. Cultures were sampled at different time points and assayed for $\beta$-galactosidase specific activity according to the method of Miller (1972). The data collected from various time points were analysed and compared by performing a two-sample independent $t$ test $(P<0.01)$ using Origin 7.0 software (Originlab Corporation). 
Biofilm formation assay. In order to determine biofilm formation on polyvinyl chloride (PVC) plastic, an assay similar to that described previously was used with a few modifications (Wei \& Zhang, 2006). Briefly, test strains were grown to saturation in LB medium and then diluted (1:100) into fresh LB broth. A $0.5 \mathrm{ml}$ volume of diluted culture was transferred to an Eppendorf tube. Bacteria were incubated without agitation for $20 \mathrm{~h}$ at $28{ }^{\circ} \mathrm{C}$ and the resulting biofilm was quantified. The biofilm was stained with $0.1 \%(\mathrm{w} / \mathrm{v})$ crystal violet for $20 \mathrm{~min}$ at room temperature and then unattached cells and residual dye were removed. Ethanol (95\%) was used to dissolve the dye that had stained the biofilm cells and the $A_{570}$ of the dissolved dye was determined.

\section{RESULTS}

\section{Identification of oprH-phoP-phoQ homologues in $P$. fluorescens 2P24}

Random mutagenesis using the transposon mini-Tn5 was performed in the reporter strain $P$. fluorescens PM101 ( $p c o I:: l a c Z$ ) to identify the potential upstream regulators of pcoI transcription. A total of 10000 inserted clones were screened on ABM plates containing kanamycin and X-Gal. Two mutants, PM400 and PM410, which formed deeper blue colonies compared with their parental strain PM101, were identified and purified for further studies. The sequences of the Tn 5 flanking DNA in both PM400 and PM410 were found to be highly similar to that of $p h o Q$, the sensor gene of the two-component regulatory system $\mathrm{PhoP} / \mathrm{PhoQ}$ found in several bacteria. This finding raised the possibility that the PhoP/PhoQ system is involved in the regulation of QS in P. fluorescens $2 \mathrm{P} 24$.

A $5.7 \mathrm{~kb}$ HindIII fragment containing the entire $p h o Q$ gene was cloned from the genomic DNA of the wild-type 2P24 (see Methods). Sequencing and BLAST analysis of this fragment revealed three ORFs (Fig. 1a), which exhibited high similarity to the oprH-phoP-phoQ locus from $P$. aeruginosa PAO1 (Macfarlane et al., 1999), and the phoPphoQ locus from S. typhimurium (Miller et al., 1989) and Escherichia coli K-12 (Kasahara et al., 1992). Identities with the $P$. aeruginosa proteins were $59 \%$ for $\mathrm{OprH}, 85 \%$ for PhoP and $68 \%$ for PhoQ. Identities with the $S$. typhimurium proteins were $53 \%$ for PhoP and $31 \%$ for PhoQ. Identities with the E. coli proteins were $53 \%$ for PhoP and $30 \%$ for PhoQ (Fig. S2). The ORFs of PhoP and PhoQ overlapped by 4 nucleotides, indicating that they could form a single transcriptional unit.

Four hexanucleotide (A/G)TTCA(G/A) direct repeats, each separated by $5 \mathrm{bp}$, were situated $61 \mathrm{bp}$ upstream of oprH (Fig. 1b). Similar direct repeats proposed to be PhoPbinding motifs have been found at a similar distance upstream of oprH-phoP-phoQ in $P$. aeruginosa $\mathrm{PAO} 1$ (Macfarlane et al., 1999).

\section{Regulation of oprH and pcol transcriptional expression by $\mathbf{M g}^{2+}$}

In $P$. fluorescens $2 \mathrm{P} 24$, the deeper blue colonies of the phoQ-defective mutants PM400 and PM410 grown on
ABM plates containing $\mathrm{X}-\mathrm{Gal}$ indicated a negative regulation of pcoI expression by PhoQ and suggested the potential involvement of $\mathrm{Mg}^{2+}$ in the regulation of the QS system. To investigate this possibility, the effect of $\mathrm{Mg}^{2+}$ on the expression of pcoI in LB medium was measured using a $p c o I$ : : lacZ transcriptional fusion in the plasmid p970-pcoI. The results revealed that pcoI transcription under low- $\mathrm{Mg}^{2+}$ conditions was significantly enhanced compared with that under high- $\mathrm{Mg}^{2+}$ conditions (Fig. 2a). Furthermore, elevated transcription of pcoI was observed when the $\mathrm{Mg}^{2+}$ concentration was changed from the high level to the low level during growth (Fig. S3). To avoid the potential influence of the anion, $\mathrm{MgSO}_{4}$ instead of $\mathrm{MgCl}_{2}$ was used to assay the $p c o I$ : : lacZ transcriptional level in LB and ABM medium (Fig. 2b, c). The results were consistent with those in $\mathrm{LB}$ medium containing $\mathrm{MgCl}_{2}$, suggesting that it was the $\mathrm{Mg}^{2+}$, and not the anion, that regulated the pcoI expression. Taken together, these results indicated that the expression of $p c o I$ is induced by the depletion of $\mathrm{Mg}^{2+}$ in P. fluorescens 2P24. Similarly, the transcription of the outer-membrane protein gene oprH, a verified $\mathrm{PhoP} /$ PhoQ-regulated gene in $P$. aeruginosa PAO1 (Macfarlane et al., 1999), was highly elevated under low- $\mathrm{Mg}^{2+}$ conditions, but was fully repressed under high- $\mathrm{Mg}^{2+}$ conditions (Fig. 2d). However, the $\mathrm{Mg}^{2+}$ signal exhibited a significantly stronger effect on $o p r H$ transcription, with an activation/repression ratio of 45 -fold; this was considerably higher than that on $p c o I$ transcription, for which the ratio was only 2 -fold. These results revealed that in strain 2P24 the transcriptions of $p c o I$ and oprH were induced by depletion of $\mathrm{Mg}^{2+}$ to a certain level.

\section{Differential regulation of oprH transcription by PhoP and PhoQ}

In order to avoid polar effects on downstream genes, inframe deletion mutants, PM402 (phoQ) and PM404 ( $p h o P$ ) (Fig. 1a), were constructed in wild-type $P$. fluorescens $2 \mathrm{P} 24$ to study the role of $\mathrm{PhoP} / \mathrm{PhoQ}$ on target genes. Under both low- and high- $\mathrm{Mg}^{2+}$ conditions, the transcription of oprH was fully repressed in the phoP deletion strain PM404 (Fig. 3), indicating that $\mathrm{PhoP}$ is a positive regulator of oprH transcription. In the phoQ-defective strain PM402, oprH maintained a similar transcription level under both growth conditions (Fig. 3), suggesting that $\mathrm{Mg}^{2+}$ loses its regulatory effect on oprH expression in the absence of PhoQ. It was interesting that, compared with the wild-type 2P24, expression of oprH in PM402 was upregulated significantly in high- $\mathrm{Mg}^{2+}$ medium but downregulated significantly in low- $\mathrm{Mg}^{2+}$ medium (Fig. 3). These results suggested that in strain $2 \mathrm{P} 24$ the transcriptional regulation of $o p r H$ by $\mathrm{PhoQ}$ is highly dependent on the environmental concentration of $\mathrm{Mg}^{2+}$. In addition, the transcription of $o p r H$ was generally maintained at a significantly higher level in PM402 (phoQ) than in PM404 (phoP), suggesting a difference in the regulation of oprH expression by the response regulator PhoP and the sensor PhoQ. 
(a) p970-pcol (pcol:::/acZ)

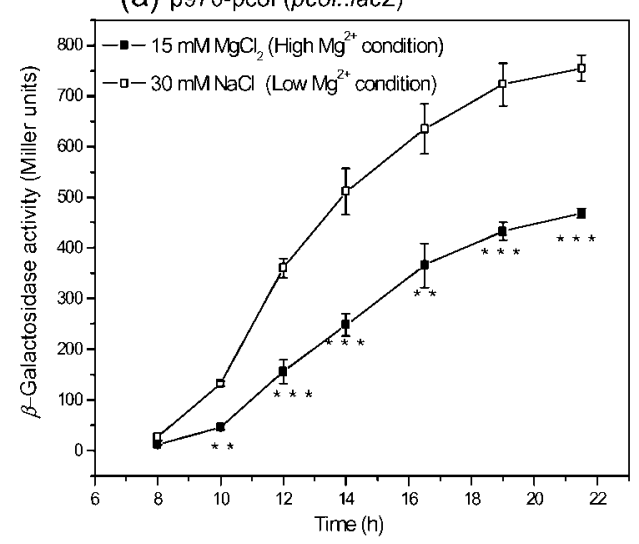

(c) p970-pcol (pcol:lacZ)

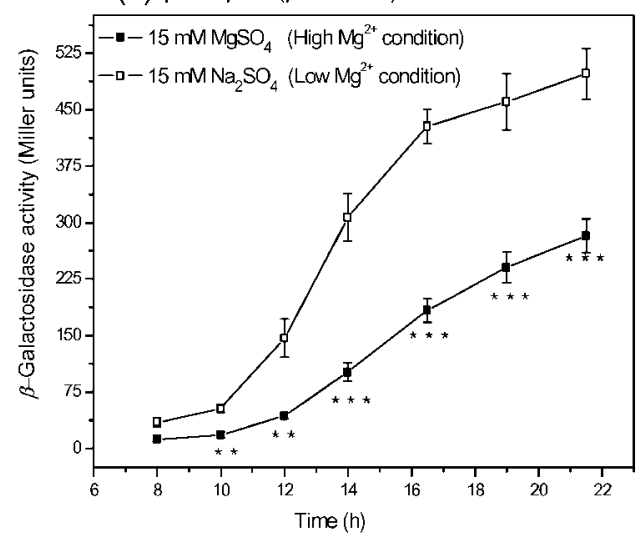

(b) p970-pcol (pcol::lacZ)

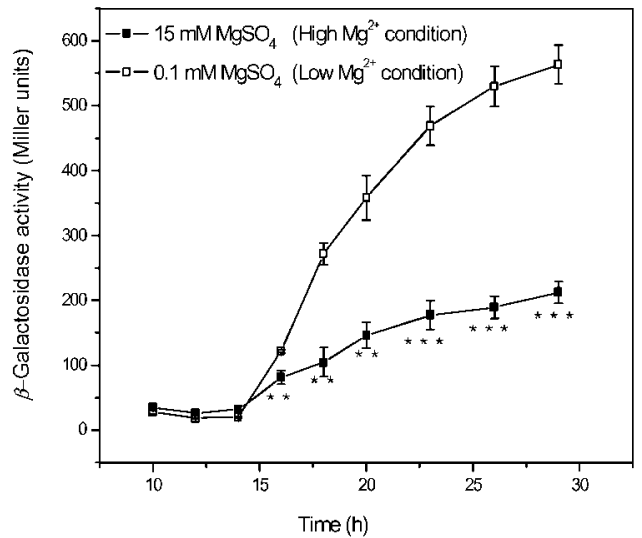

(d) $\mathrm{p} 970-\mathrm{H}$ (oprH:JacZ)

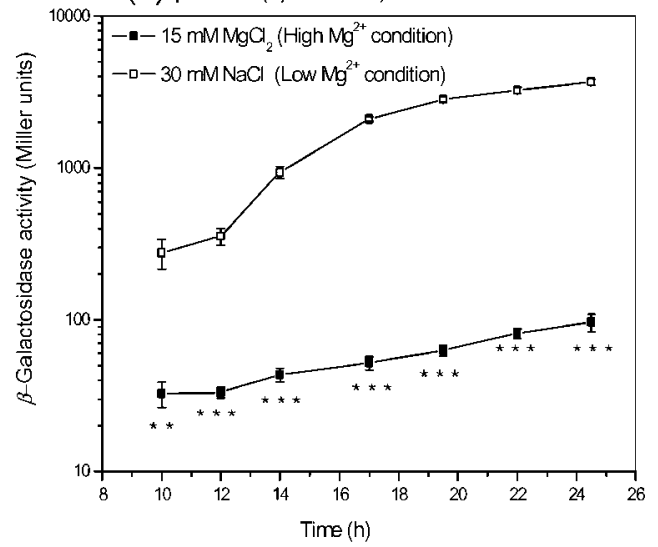

Fig. 2. $\mathrm{Mg}^{2+}$ regulates $\mathrm{pcol}$ and $\mathrm{oprH}$ expression in $P$. fluorescens $2 \mathrm{P} 24 . \quad \beta$-Galactosidase activity of the plasmid transcriptional fusion lac $Z$ in the wild-type 2P24 was measured at various time points after inoculation into LB medium (a, c, d) and $\mathrm{ABM}$ medium (b) containing high or low $\mathrm{Mg}^{2+}$. All experiments were performed in triplicate; means $\pm \mathrm{SD}$ are plotted. Statistical analyses were performed with the two-sample independent $t$ test. ${ }^{\star \star}, P<0.01 ;{ }^{\star \star \star}, P<0.001$.
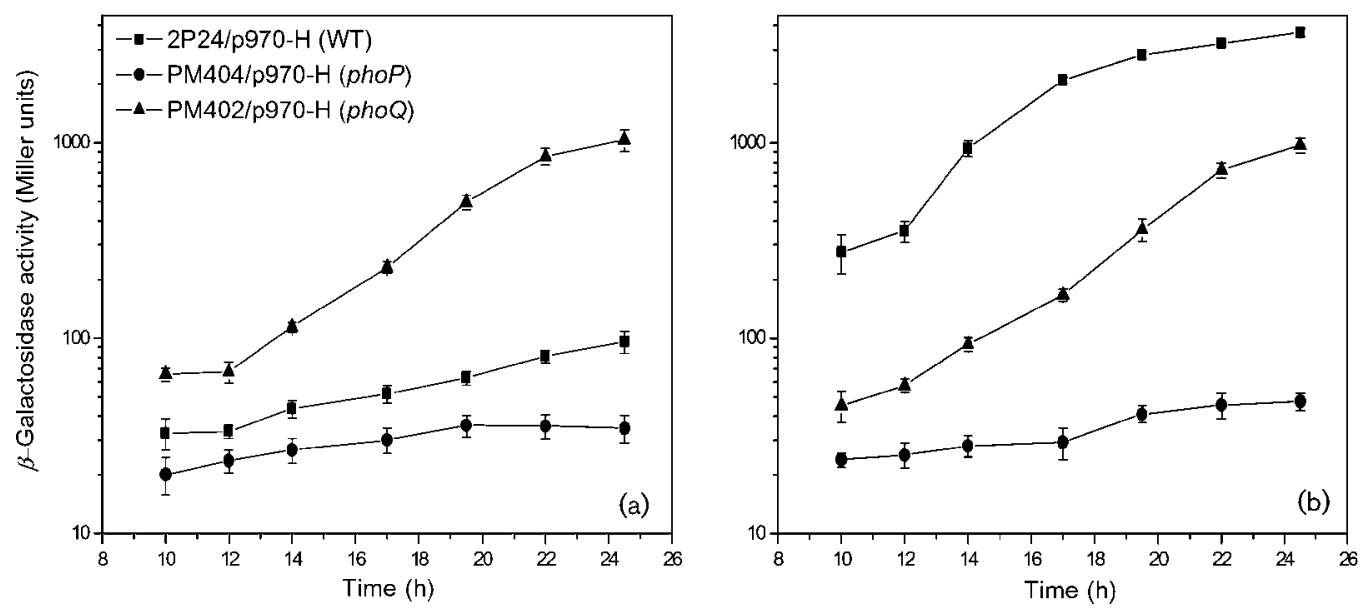

Fig. 3. $\mathrm{Mg}^{2+}$ regulates oprH expression in a PhoP/PhoQ-dependent manner. $\beta$-Galactosidase activity of the plasmid p970-H (oprH: : lacZ) in P. fluorescens strains was measured at various time points after inoculation into LB medium. All experiments were performed in triplicate; means \pm SD are plotted. (a) High- $\mathrm{Mg}^{2+}$ condition: LB medium containing $15 \mathrm{mM} \mathrm{MgCl}_{2}$. (b) Low$\mathrm{Mg}^{2+}$ condition: LB medium containing $30 \mathrm{mM} \mathrm{NaCl}$. 


\section{Regulation of QS by the two-component PhoP/ PhoQ system}

In order to determine the potential regulatory effect of the PhoP/PhoQ system on pcoI expression, the $\beta$ galactosidase activity of a genomic $p c o I$ : : lac $Z$ transcriptional fusion was measured in the mutants PM401 (phoQ, pcoI::lacZ) and PM403 (phoP, pcoI::lacZ), and also in their parental strain PM101 (pcoI::lacZ). The expression of $p c o I:$ : lac $Z$ was elevated significantly in the phoQ deletion strain PM401 under both low- and high$\mathrm{Mg}^{2+}$ conditions. Introduction of a $p h o Q^{+}$plasmid, p415-phoQ, into strain PM401 restored the pcoI:: lacZ activity to the wild-type level (Fig. 4), suggesting that PhoQ acts as a negative regulator on pcoI expression. In contrast, when the bacteria were grown in high- $\mathrm{Mg}^{2+}$ medium, there was no significant difference in the pcoI:: lac $Z$ activity between the phoP deletion strain PM403 and its wild-type PM101. The pcoI: : lacZ activity, however, was significantly suppressed in PM403 under low- $\mathrm{Mg}^{2+}$ conditions (Fig. 4b). Introduction of a $p h o P^{+}$ plasmid, p415-phoP, into strain PM403 resulted in a slight overexpression of $p c o I:$ : lac $Z$ in high- $\mathrm{Mg}^{2+}$ medium, which was considerably greater than that in low$\mathrm{Mg}^{2+}$ medium (Fig. 4). These observations indicated that PhoP positively regulates pcoI expression and that this regulation is more pronounced under low- $\mathrm{Mg}^{2+}$ conditions.

The accumulation of QS signals (AHL) in P. fluorescens $2 \mathrm{P} 24$ and its derivatives was measured and compared quantitatively using the biosensor strain A. tumefaciens NTL4(pZLR4). The result (Fig. 5) was consistent with the pcoI:: lacZ transcriptional assay described above and confirmed the involvement of the PhoP/PhoQ system in the regulation of the QS system in strain 2P24.

\section{Effect of PhoP/PhoQ on biofilm formation}

Since the PhoP/PhoQ system is involved in the regulation of pcoI transcription in strain $2 \mathrm{P} 24$, we reasoned that the characteristics controlled by the QS system might be also influenced by PhoP/PhoQ. Biofilm formation on PVC plastic is known to be positively controlled by QS in strain 2P24 (Wei \& Zhang, 2006). Therefore, an experiment was performed to examine whether biofilm formation was affected by $\mathrm{PhoP} / \mathrm{PhoQ}$. When cultured in high- $\mathrm{Mg}^{2+}$ medium (Fig. 6), neither the phoP nor the phoQ mutant caused a significant change in the biofilm formation in Eppendorf tubes. In contrast, when cultured in low- $\mathrm{Mg}^{2+}$ medium, mutant PM402 (phoQ) exhibited a significantly enhanced biofilm formation, and this mutation could be restored by the plasmid p415-phoQ $\left(p h o Q^{+}\right)$. Mutation of phoP, however, had no significant influence on biofilm formation. These observations indicated the negative role of PhoQ in biofilm formation under the low- $\mathrm{Mg}^{2+}$ condition; however, this regulation did not appear to be mediated via the regulator PhoP.

\section{Effect of PhoP/PhoQ on bacterial colony formation}

When grown on LB plates, the phoQ mutant PM402 formed significantly smaller colonies compared with the wild-type under both low- and high- $\mathrm{Mg}^{2+}$ conditions. In contrast, the phoP mutant PM404 formed larger colonies on both media (Fig. 7). Introduction of the complementary plasmids p415-phoQ and p415-phoP, respectively, restored the colony size to the wild-type level. Soncini et al. (1996) reported that the mutation of phoP resulted in a larger cell size in S. typhimurium. However, no such morphological change was observed in the phoP or phoQ mutant of $2 \mathrm{P} 24$ (data not shown). The number of living cells from
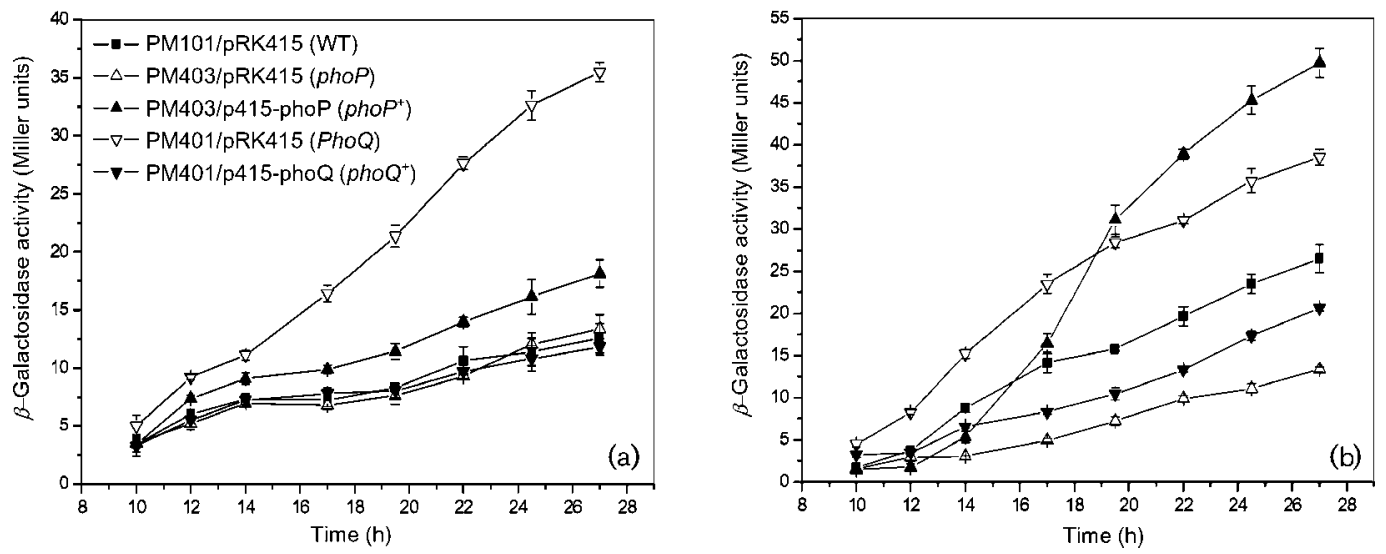

Fig. 4. Regulation of the transcriptional expression of $p c o l$ by PhoP/PhoO. $\beta$-Galactosidase activity of the genomic fusion pcol: : lacZ in PM101 and it derivatives was measured at various time points after inoculation into LB medium. All experiments were performed in triplicate; means $\pm \mathrm{SD}$ are plotted. (a) High- $\mathrm{Mg}^{2+}$ condition: $\mathrm{LB}$ medium containing $15 \mathrm{mM} \mathrm{MgCl}$. (b) Low$\mathrm{Mg}^{2+}$ condition: LB medium containing $30 \mathrm{mM} \mathrm{NaCl}$. 


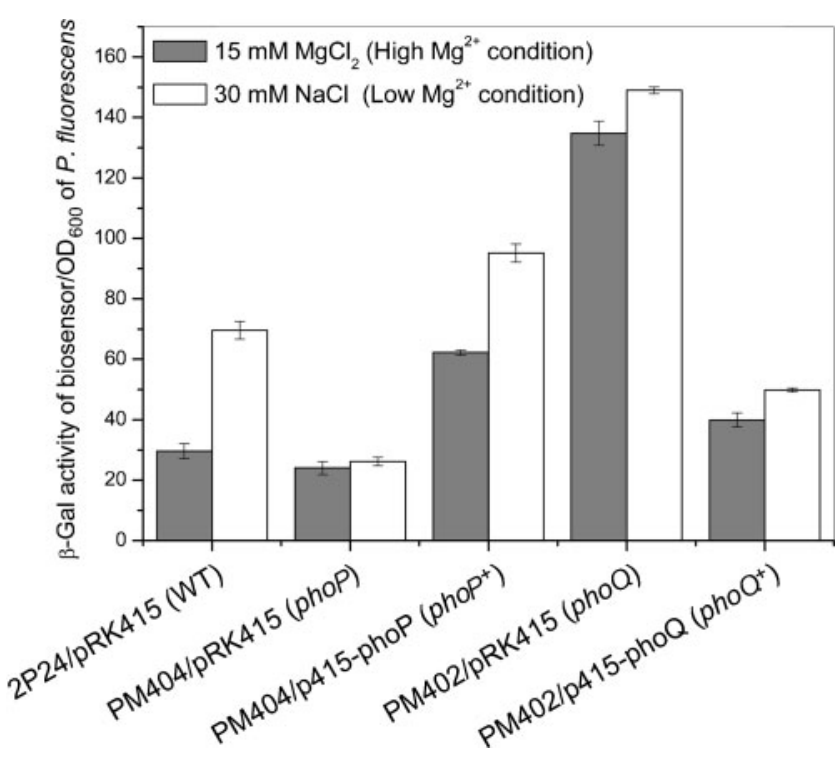

Fig. 5. Regulation of $A H L$ production by PhoP/PhoO. $\beta$ Galactosidase activity of the traG: : lac Z fusion in the biosensor strain A. tumefaciens NTL4(pZLR4) was measured after incubation with $\mathrm{AHL}$ that had been extracted from the wild-type 2P24 and its derivatives. The cell density $\left(\mathrm{OD}_{600}\right)$ of each strain was measured. All experiments were performed in triplicate; means \pm SD are plotted.

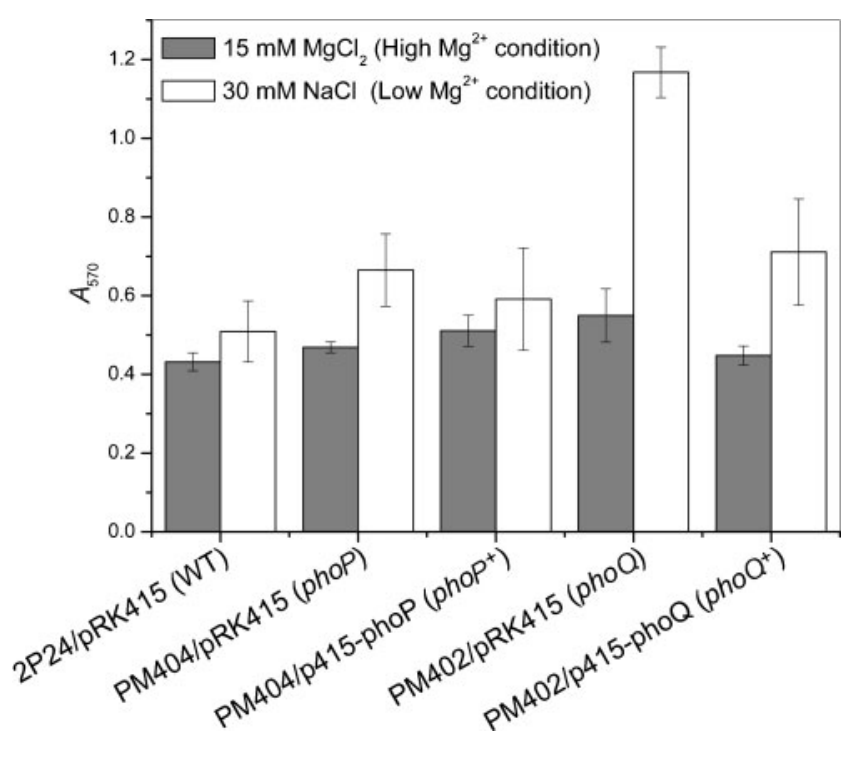

Fig. 6. Regulation of biofilm formation by PhoP/PhoQ. A biofilm was allowed to form in Eppendorf tubes. The tubes were incubated for $20 \mathrm{~h}$ at $28^{\circ} \mathrm{C}$. The unattached bacteria were rinsed off and the biofilm was stained with crystal violet. The residual dye was dissolved and the $A_{570}$ of the solution was determined. All experiments were performed in triplicate; means \pm SD are plotted. individual colonies was checked by plating and it was found that the number of such cells in colonies of the $p h o Q$ mutant $\left(2.5 \times 10^{6}\right.$ cells per colony) was considerably less than that in the wild-type $2 \mathrm{P} 24\left(1.9 \times 10^{8}\right.$ cells per colony $)$, indicating that the different colony sizes might be a result of differences in cell population. This result is consistent with the slower growth rate of the phoQ mutant in LB broth under both low- and high- $\mathrm{Mg}^{2+}$ conditions (Fig. S4). Additionally, the changes in colony size of the PhoP/ PhoQ mutants were unlikely to have been caused by $\mathrm{Mg}^{2+}$ starvation in the growth media, since similar regulation of colony size by PhoP/PhoQ was observed when the mutant cells were cultured in high- $\mathrm{Mg}^{2+}$ medium, although the bacterial colonies of 2P24 strains were generally smaller in low- $\mathrm{Mg}^{2+}$ medium.

\section{DISCUSSION}

The PhoP/PhoQ locus identified in P. fluorescens strain $2 \mathrm{P} 24$ has the highest similarity to that in $P$. aeruginosa $\mathrm{PAO} 1$, in which the expression of the outer-membrane protein $\mathrm{OprH}$ is regulated by environmental $\mathrm{Mg}^{2+}$ signals via the PhoP/PhoQ system (Macfarlane et al., 1999). Similarly, the transcriptional expression of oprH was also induced by $\mathrm{Mg}^{2+}$ starvation in wild-type 2P24 (Fig. 1b), indicating that $\mathrm{Mg}^{2+}$ concentration is an environmental cue for oprH expression in strain 2P24. But the expression of oprH was not changed in the phoP or phoQ mutant, irrespective of the $\mathrm{Mg}^{2+}$ level in the medium (Fig. 3). These observations suggest that the presence of both PhoP and $\mathrm{PhoQ}$ is necessary for the $\mathrm{Mg}^{2+}$ regulation of oprH expression. In strain $2 \mathrm{P} 24$, PhoP functions as a positive regulator of oprH expression under both low- and high$\mathrm{Mg}^{2+}$ conditions. This observation is consistent with the results of a Western blot analysis of OprH expression in $P$. aeruginosa PAO1 (Macfarlane et al., 1999). In strain 2P24, however, the sensor PhoQ plays opposite roles in oprH transcription in response to changes in $\mathrm{Mg}^{2+}$ level in the growth medium (Fig. 3). Compared with the wild-type strain, oprH expression in the phoQ mutant was downregulated in low- $\mathrm{Mg}^{2+}$ medium but upregulated in high$\mathrm{Mg}^{2+}$ medium. This result differed from the expression of OprH observed in $P$. aeruginosa PAO1, which was consistently activated in the absence of PhoQ in both low- and high- $\mathrm{Mg}^{2+}$ media (Macfarlane et al., 1999). Given the fact that the active form of response regulators is usually the phosphorylated form, our results support the hypothesis that in strain 2P24, PhoQ acts as a kinase to activate PhoP under low- $\mathrm{Mg}^{2+}$ conditions but acts as a phosphatase to repress PhoP under high- $\mathrm{Mg}^{2+}$ conditions. This is consistent with an in vitro assay indicating that phosphatase activity is the target for $\mathrm{Mg}^{2+}$ regulation of the sensor PhoQ in S. typhimurium (Castelli et al., 2000). However, this hypothesis does not adequately address the observation that oprH transcription is maintained at a higher level in the phoQ mutant than in the phoP mutant under both low- and high- $\mathrm{Mg}^{2+}$ conditions, which 


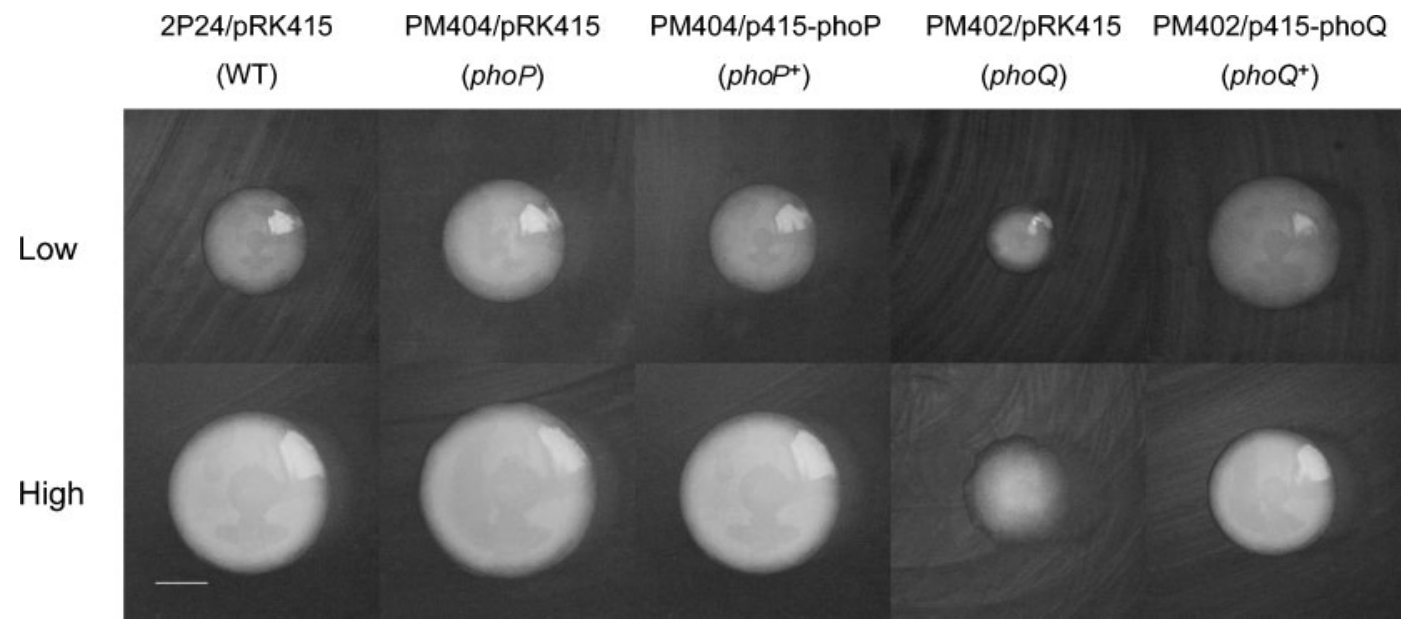

Fig. 7. Regulation of colony formation by PhoP/PhoQ. The wild-type strain $2 \mathrm{P} 24$ and its derivatives were cultured on LB plates for 3 days at $28{ }^{\circ} \mathrm{C}$. High, high- $\mathrm{Mg}^{2+}$ condition: LB medium containing $15 \mathrm{mM} \mathrm{MgCl}$. Low, low- $\mathrm{Mg}^{2+}$ condition: LB medium containing $30 \mathrm{mM} \mathrm{NaCl}$. Bar, $1 \mathrm{~mm}$.

indicates that PhoP alone is capable of activating the transcription of $o p r H$. A possible explanation for this observation is that one or more alternative kinases present in strain 2P24 mediate the PhoP phosphorylation in the PhoQ-negative background. It is well known that twocomponent signal-transduction systems are often linked. In E. coli strain MG1655, EvgS/EvgA regulates PhoP/PhoQ through a small membrane protein B1500 (Eguchi et al., 2007). Moreover, Matsubara et al. (2000) described a crossphosphorylation between $\mathrm{ArcA} / \mathrm{ArcB}$ and EnvZ/OmpR in E. coli K-12. Further experiments are needed to determine the phosphorylation mechanism of the response regulator PhoP in strain $2 \mathrm{P} 24$.

Our genetic evidence suggests that the PhoP/PhoQ twocomponent system acts as a new regulator of QS in strain $P$. fluorescens 2P24. Two-component regulatory systems represent one of the most common mechanisms of transmembrane signal transduction in bacteria. The QS signalling in many Gram-negative bacteria is often subservient to the two-component system, to connect with environmental signals. In $P$. aeruginosa strain SCV20265, phosphate limitation activates the $r h l \mathrm{QS}$ system through the PhoB/PhoR two-component system (Jensen et al., 2006); the global two-component system GacS/GacA, which responds to an unknown signal, regulates the $r h l$ and las QS system in P. aeruginosa (Reimmann et al., 1997) and the pco QS system in P. fluorescens 2P24 (Yan et al., 2008). The regulation of $p c o I$ expression by $\mathrm{PhoP} / \mathrm{PhoQ}$ described in this study linked $\mathrm{Mg}^{2+}$ signal with the $p c o \mathrm{QS}$ system in strain $2 \mathrm{P} 24$, and reflected the complex mechanism of QS, which not only responded to the cell density but also connected with various environmental stimuli.

The deletion and complementation experiments in this study demonstrated that PhoP positively regulates both pcoI transcription (Fig. 4) and AHL production (Fig. 5).
Moreover, the regulatory effect was more prominent under low- $\mathrm{Mg}^{2+}$ conditions. Similar to the regulation of oprH transcription, the phoQ mutant exhibited a consistently higher level of pcoI transcription than that in the phoP mutant under both low- and high- $\mathrm{Mg}^{2+}$ conditions, which also supports the existence of a second phosphorylation agent for PhoP. However, unlike the opposite regulatory effect of PhoQ on oprH in response to different $\mathrm{Mg}^{2+}$ conditions, PhoQ merely acts as a negative regulator of pcoI transcription irrespective of the $\mathrm{Mg}^{2+}$ level in the medium (Fig. 4). These findings demonstrate a clearly different regulation of downstream phenotypes by the $\mathrm{PhoP} / \mathrm{PhoQ}$ system in 2P24, and reflect the complexity of the signal transduction pathway mediated by the PhoP/ PhoQ system in this strain.

The response regulator PhoP has been demonstrated to directly activate many genes by binding with direct repeats upstream of the target genes in E. coli strains K-12 (Kato et al., 1999) and MC4100 (Minagawa et al., 2003) and in $P$. aeruginosa PAO1 (McPhee et al., 2006), and also appears to directly activate the expression of oprH in strain 2P24 by binding with similar direct hexanucleotide repeats upstream of oprH (Fig. 1b). PhoP was also found to positively regulate pcoI transcription under low- $\mathrm{Mg}^{2+}$ conditions, but compared with the 77 -fold reduction in oprH expression caused by the phoP mutation (Fig. 3b), only a 3-fold reduction of pcoI expression was observed (Fig. 4b). These observations indicate different regulatory mechanisms of PhoP on the expression of oprH and pcoI. Combined with the observation that there is no similar binding motif located around the putative promoter of pcoI (Wei \& Zhang, 2006), we propose that PhoP regulates pcoI expression through an indirect pathway.

The QS systems LasI/LasR and RhlI/RhlR play important roles in biofilm formation in $P$. aeruginosa PA14 by 
regulating the expression of pel genes (Sakuragi \& Kolter, 2007). Similarly, the pcoI gene was previously demonstrated to positively influence biofilm formation in strain 2P24 (Wei \& Zhang, 2006). The biofilm assay performed in this study demonstrated that biofilm formation in $2 \mathrm{P} 24$ is negatively regulated by $\mathrm{PhoQ}$ in low- $\mathrm{Mg}^{2+}$ medium, but that this regulation is independent of $\mathrm{PhoP}$ since no significant influence was observed following phoP deletion or $\mathrm{Mg}^{2+}$ depletion (Fig. 6). This observation is contrary to the regulatory model of QS by PhoP/PhoQ described in this paper, indicating that the influence of PhoQ on biofilm formation was probably not via the QS system and that, in 2P24, PhoQ probably acts through a response regulator(s) other than PhoP. In contrast to the situation in $2 \mathrm{P} 24$, a dynamic biofilm assay in $P$. aeruginosa PA14 demonstrated that PhoQ positively regulates the biofilm formation (Ramsey \& Whiteley, 2004). Additionally, a phoP-insertion mutant in S. typhimurium resulted in the earlier maturation of biofilms on gallstones and enhanced biofilm formation on glass (Prouty \& Gunn, 2003). The differential regulation of biofilms by PhoP/PhoQ may imply that different strains have evolved fine-tuned regulatory features of PhoP/PhoQ to adapt to and enhance colonization in particular niches and environments.

\section{ACKNOWLEDGEMENTS}

This work was funded by the National Natural Science Foundation of China (30671403, 30871666), National Programs for High Technology Research and Development of China (2006AA10A211) and the Chinese MOST-DEST Cooperation Project (2007DFA31570).

\section{REFERENCES}

Bertani, I. \& Venturi, V. (2004). Regulation of the $N$-acyl homoserine lactone-dependent quorum-sensing system in rhizosphere Pseudomonas putida WCS358 and cross-talk with the stationaryphase RpoS sigma factor and the global regulator GacA. Appl Environ Microbiol 70, 5493-5502.

Brinkman, F. S., Macfarlane, E. L., Warrener, P. \& Hancock, R. E. (2001). Evolutionary relationships among virulence-associated histidine kinases. Infect Immun 69, 5207-5211.

Castelli, M. E., Ve'scovi, E. G. \& Soncini, F. C. (2000). The phosphatase activity is the target for $\mathrm{Mg}^{2+}$ regulation of the sensor protein PhoQ in Salmonella. J Biol Chem 275, 22948-22954.

Cha, C., Gao, P., Chen, Y. C., Shaw, P. D. \& Farrand, S. K. (1998). Production of acyl-homoserine lactone quorum-sensing signals by Gram-negative plant-associated bacteria. Mol Plant Microbe Interact 11, 1119-1129.

Chilton, M. D., Currier, T. C., Farrand, S. K., Bendich, A. J., Gordon, M. P. \& Nester, E. W. (1974). Agrobacterium tumefaciens DNA and PS8 bacteriophage DNA not detected in crown gall tumors. Proc Natl Acad Sci U S A 71, 3672-3676.

de Kievit, T. R. \& Iglewski, B. H. (2000). Bacterial quorum sensing in pathogenic relationships. Infect Immun 68, 4839-4849.

Eguchi, Y., Itou, J., Yamane, M., Demizu, R., Yamato, F., Okada, A., Mori, H., Kato, A. \& Utsumi, R. (2007). B1500, a small membrane protein, connects the two-component systems EvgS/EvgA and PhoQ/ PhoP in Escherichia coli. Proc Natl Acad Sci U S A 104, 18712-18717.
Ernst, R. K., Yi, E. C., Guo, L., Lim, K. B., Burns, J. L., Hackett, M. \& Miller, S. I. (1999). Specific lipopolysaccharide found in cystic fibrosis airway Pseudomonas aeruginosa. Science 286, 1561-1565.

González, J. E. \& Keshavan, N. D. (2006). Messing with bacterial quorum sensing. Microbiol Mol Biol Rev 70, 859-875.

Groisman, E. A. (2001). The pleiotropic two-component regulatory system PhoP-PhoQ. J Bacteriol 183, 1835-1842.

Herrero, M., Lorenzo, V. D. \& Timmis, K. N. (1990). Transposon vectors containing non-antibiotic resistance selection markers for cloning and stable chromosomal insertion of foreign genes in gramnegative bacteria. J Bacteriol 172, 6557-6567.

Jensen, V., Löns, D., Zaoui, C., Bredenbruch, F., Meissner, A., Dieterich, G., Münch, R. \& Häussler, S. (2006). RhlR expression in Pseudomonas aeruginosa is modulated by the Pseudomonas quinolone signal via PhoB-dependent and -independent pathways. J Bacteriol 188, 8601-8606.

Juhas, M., Wiehlmann, L., Huber, B., Jordan, D., Lauber, J., Salunkhe, P., Limpert, A. S., von Gǒta, F., Steinmetz, I. \& other authors (2004). Global regulation of quorum sensing and virulence by VqsR in Pseudomonas aeruginosa. Microbiology 150, 831-841.

Kasahara, M., Nakata, A. \& Shinagawa, H. (1992). Molecular analysis of the Escherichia coli PhoP-PhoQ operon. J Bacteriol 174, 492-498.

Kato, A., Tanabe, H. \& Utsumi, R. (1999). Molecular characterization of the PhoP-PhoQ two-component system in Escherichia coli K-12: identification of extracellular $\mathrm{Mg}^{2+}$-responsive promoters. J Bacteriol 181, 5516-5520.

Keen, N. T., Tamaki, S., Kobayashi, D. \& Trollinger, D. (1988). Improved broad-host-range plasmids for DNA cloning in gramnegative bacteria. Gene 70, 191-197.

Keller, L. \& Surette, M. G. (2006). Communication in bacteria: an ecological and evolutionary perspective. Nat Rev Microbiol 4, 249258.

Kier, L. D., Weppelman, R. M. \& Ames, B. N. (1979). Regulation of nonspecific acid phosphatase in Salmonella: phoN and phoP genes. $J$ Bacteriol 138, 155-161.

Macfarlane, E. L., Kwasnicka, A., Ochs, M. M. \& Hancock, R. E. (1999). PhoP-PhoQ homologues in Pseudomonas aeruginosa regulate expression of the outer-membrane protein OprH and polymyxin B resistance. Mol Microbiol 34, 305-316.

Macfarlane, E. L. A., Kwasnicka, A. \& Hancock, R. E. W. (2000). Role of Pseudomonas aeruginosa in resistance to antimicrobial peptides and aminoglycosides. Microbiology 146, 2543-2554.

Matsubara, M., Kitaota, S., Takeda, S. \& Mizuno, T. (2000). Tuning of the porin expression under anaerobic growth conditions by His-toAsp cross-phosphorelay through both the EnvZ-osmosensor and ArcB-anaerosensor in Escherichia coli. Genes Cells 5, 555-569.

McPhee, J. B., Bains, M., Winsor, G., Lewenza, S., Kwasnicka, A., Brazas, M. D., Brinkman, F. S. \& Hancock, R. E. (2006). Contribution of the PhoP-PhoQ and PmrA-PmrB two-component regulatory systems to $\mathrm{Mg}^{2+}$-induced gene regulation in Pseudomonas aeruginosa. J Bacteriol 188, 3995-4006.

Miller, J. H. (1972). Experiments in Molecular Genetics. Cold Spring Harbor, NY: Cold Spring Harbor Laboratory.

Miller, M. B. \& Bassler, B. L. (2001). Quorum sensing in bacteria. Annu Rev Microbiol 55, 165-199.

Miller, S. I., Kukral, A. M. \& Mekalanos, J. J. (1989). A two-component regulatory system ( $p h o P$ phoQ) controls Salmonella typhimurium virulence. Proc Natl Acad Sci U S A 86, 5054-5058.

Minagawa, S., Ogasawara, H., Kato, A., Yamamoto, K., Eguchi, Y., Oshima, T., Mori, H., Ishihama, A. \& Utsumi, R. (2003). Identification 
and molecular characterization of the $\mathrm{Mg}^{2+}$ stimulon of Escherichia coli. J Bacteriol 185, 3696-3702.

Pessi, G., Williams, F., Hindle, Z., Heurlier, K., Holden, M. T., Camara, M., Haas, D. \& Williams, P. (2001). The global posttranscriptional regulator RsmA modulates production of virulence determinants and $\mathrm{N}$-acylhomoserine lactones in Pseudomonas aeruginosa. J Bacteriol 183, 6676-6683.

Prouty, A. M. \& Gunn, J. S. (2003). Comparative analysis of Salmonella enterica serovar Typhimurium biofilm formation on gallstones and on glass. Infect Immun 71, 7154-7158.

Rampioni, G., Polticelli, F., Bertani, I., Righetti, K., Venturi, V., Zennaro, E. \& Leoni, L. (2007). The Pseudomonas quorum-sensing regulator RsaL belongs to the tetrahelical superclass of $\mathrm{H}-\mathrm{T}-\mathrm{H}$ proteins. J Bacteriol 189, 1922-1930.

Ramsey, M. M. \& Whiteley, M. (2004). Pseudomonas aeruginosa attachment and biofilm development in dynamic environments. Mol Microbiol 53, 1075-1087.

Reimmann, C., Beyeler, M., Latifi, A., Winteler, H., Foglino, M., Lazdunski, A. \& Haas, D. (1997). The global activator GacA of Pseudomonas aeruginosa PAO1 positively controls the production of the autoinducer $\mathrm{N}$-butyryl-homoserine lactone and the formation of the virulence factors pyocyanin, cyanide, and lipase. Mol Microbiol 24, 309-319.

Sakuragi, Y. \& Kolter, R. (2007). Quorum-sensing regulation of the biofilm matrix genes (pel) of Pseudomonas aeruginosa. J Bacteriol 189, 5383-5386.

Sambrook, J., Fritsch, E. F. \& Maniatis, T. (1989). Molecular Cloning: a Laboratory Manual, 2nd edn. Cold Spring Harbor, NY: Cold Spring Harbor Laboratory.
Soncini, F. C., Véscovi, E. G., Solomon, F. \& Groisman, E. A. (1996). Molecular basis of the magnesium deprivation response in Salmonella typhimurium: identification of PhoP-regulated genes. J Bacteriol 178, 5092-5099.

Van den Eede, G., Deblaere, R., Goethals, K., Van Montagu, M. \& Holsters, M. (1992). Broad host range and promoter selection vectors for bacteria that interact with plants. Mol Plant Microbe Interact 5, 228-234.

Venturi, V. (2006). Regulation of quorum sensing in Pseudomonas. FEMS Microbiol Rev 30, 274-291.

Véscovi, E. G., Soncini, F. C. \& Groisman, E. G. (1996). $\mathrm{Mg}^{2+}$ as an extracellular signal: environmental regulation of Salmonella virulence. Cell 84, 165-174.

Waters, C. M., Lu, W., Rabinowitz, J. D. \& Bassler, B. L. (2008). Quorum sensing controls biofilm formation in Vibrio cholerae through modulation of cyclic di-GMP levels and repression of $v p s T$. J Bacteriol 190, 2527-2536.

Wei, H. L. \& Zhang, L. Q. (2005). Cloning and functional characterization of the gacS gene of the biocontrol strain Pseuodomonas fluorescens 2P24. Wei Sheng Wu Xue Bao 45, 368-372.

Wei, H. L. \& Zhang, L. Q. (2006). Quorum-sensing system influences root colonization and biological control ability in Pseudomonas fluorescens 2P24. Antonie Van Leeuwenhoek 89, 267-280.

Yan, Q., Wu, X. G., Wei, H. L., Wang, H. M. \& Zhang, L. Q. (2008). Differential control of the PcoI-PcoR quorum-sensing system in Pseudomonas fluorescens 2P24 by sigma factor RpoS and the GacS/ GacA two-component regulatory system. Microbiol Res, doi:10.1016/ j.micres.2008.02.001

Edited by: C. Boucher 